\title{
Ex-tauglicher Kompakt-Raman-Sensor für das Qualitätsmonitoring in der chemischen Industrie
}

\author{
M. Nachtmann 1 , F. Braun ${ }^{1}$, S. Keck' , H.-S. Eckhardt ${ }^{2}$, C. Mattolat ${ }^{2}$, N. Gretz ${ }^{3}$, S. Scholl ${ }^{4}$, M. Rädle ${ }^{1}$ \\ ${ }^{1}$ Hochschule Mannheim, Institut für Prozessmesstechnik und Innovative Energiesysteme, Paul- \\ Wittsack-Str. 10, 68163 Mannheim, Deutschland; \\ 2tec5 AG, In der Au 2761440 Oberursel/Ts, Deutschland \\ ${ }^{3}$ Universität Heidelberg, Zentrum für Medizinische Forschung, Medizinische Fakultät Mannheim, \\ Theodor-Kutzer-Ufer 1-3, 68167 Mannheim, Deutschland \\ ${ }^{4}$ Technische Universität Braunschweig, Institut für Chemische und Thermische Verfahrenstechnik, \\ Langer Kamp 7, 38106 Braunschweig, Deutschland \\ m.nachtmann@hs-mannheim.de
}

\begin{abstract}
Zusammenfassung
In diesem Beitrag wird die Entwicklung eines photometrischen Konzepts zur Prozesskontrolle auf Grundlage des Raman-Effekts vorgestellt. Raman-Spektrometer erhalten als Werkzeug im Rahmen des molekülspezifischen Konzentrationsmonitorings immer stärker Einzug in die Prozessanalysentechnik. Allerdings ist neben den benötigten Laserleistungen, die eine Verwendung in explosionsgefährdeten Bereichen einschränken, auch die finanzielle Einstiegshürde hoch. Es wird im Rahmen eines Proof of Concept gezeigt, dass photometrische Messungen der Raman-Streuung möglich sind. Durch den Einsatz hochsensitiver Detektoren kann eine Reduktion der benötigten Anregungsleistung erreicht werden, um einen Einsatz in Ex-Zonen zu realisieren. Bauartbedingt benötigen Photometer, verglichen mit Spektrometer-Systemen, weniger aufwändige Optikaufbauten. Dies ermöglicht eine deutliche Reduktion der Kosten. Die Integration einer Embedded-Plattform ermöglicht den Einsatz als autonomen Sensor, da das Photometer intern alle Berechnungen durchführt und direkt an die Prozesskommunikation angebunden wird.
\end{abstract}

Keywords: Raman-Spektroskopie,optische Messverfahren,Photometer,ATEX,EmbeddedProzessanalysentechnik (PAT)

\section{Einleitung}

Molekülspezifisches Konzentrationsmonitoring basierend auf dem Raman-Effekt hält als InlineWerkzeug der Prozessanalysentechnik (PAT) Einzug in immer mehr Bereiche der chemischen Industrie. Bisher wird dieser Bedarf vorwiegend durch Spektrometer-Systeme bedient und Spektren über den kompletten Messbereich aufgenommen. Die Fülle an spektralen Informationen eines Spektrometers wird jedoch nicht in allen Fällen benötigt. Im Bereich der UVNIS-/NIR- und Fluoreszenz-Spektroskopie haben sich aus diesem Grund neben Spektrometer-Systemen auch photometrische Konzepte etabliert. Diese reduzieren die Datenmenge erheblich, da nur für den Prozess spezifische Banden detektiert und z.B. an ein Prozessleitsystem (PLS) weitergeleitet werden. In diesem Beitrag werden die Entwicklung eines Raman-Photometers für explosionsgefährdete Umgebungen sowie einige Anwendungsgebiete skizziert. Ein besonderer Fokus liegt hierbei auf der Auswahl und Integration der verwendeten Sensoren sowie dem GesamtSensorkonzept [1,2].

\section{Stand der Technik}

Die Entwicklung und Herstellung kostengünstiger Raman-Sensoren ist seit einiger Zeit das Bestreben der analytischen Industrie. Im Laufe der Zeit haben sich einige Patente in Bezug auf ein Laser- und ein RamanPhotometer im speziellen angesammelt. Hier soll ein kurzer Überblick gegeben werden.

Ein Patent aus dem Jahr 2008 (US20080180661) der Firma Hamilton Sundstrand beschreibt ein Raman-Photometer für die industrielle Prozesskontrolle. Dieses ist als einziges Produkt auf dem Markt erhältlich. Es wird unter dem Namen „RPM ${ }^{\circledR}$ View“ vertrieben. Das Patent beschreibt einige Möglichkeiten für den Aufbau und die Konstruktion eines Photometers. Ein Hauptmerkmal des Systems bildet ein rotierendes 
Filterrad. In diesem sind mehrere unterschiedliche Filter angebracht, die eine sequentielle Messung bei verschiedenen Wellenlängen garantieren. Hiermit ist es möglich, verschiedene Peaks zu verfolgen bzw. eine Referenzmessung durchzuführen. Allerdings steht ein Filterrad einer simultanen Messung von Referenz- und Messsignal im Wege. Bei chemischen Reaktionen können sich Prozessparameter innerhalb kürzester Zeit ändern, daher ist ein zeitlicher Versatz nicht zielführend und verfälscht die Messung entscheidend. Bei dem in diesem Beitrag vorgestellten optischen Aufbau wird ein anderer Weg gewählt, besonders um simultane Messungen des Analyten und der Referenzen zu gewährleisten [5].

Das Patent (DE000002103318B) aus dem Jahr 1970 beschäftigt sich mit dem grundlegenden Aufbau eines Streulicht-Photometers. Ein kollimierter Laser wird mittels optischen Linsen durch eine Blende auf eine Probe fokussiert. Diese trennt die in einem bestimmten Winkel gestreute Strahlung über eine weitere Blende von Störlicht. Eine optische Linse fokussiert das Licht auf eine weitere Blende. Eine KollimatorLinse leitet die gestreute elektromagnetische Strahlung auf eine Detektoreinheit. Der Aufbau ist für einen definierten Streuwinkel ausgelegt. Der Raman-Effekt tritt allerdings in allen Raumrichtungen auf [6].

Weiterhin gibt es Entwicklungen, die sich mit einem Spektrophotometer-Ansatz dem Thema annähern. Dies wird in einer Veröffentlichung von B. Berenblut aus dem Jahre 1971 mit dem Titel „The modificaction of a Cary model 81 Raman spectrophotometer for use with a laser" beschrieben [4]. Bei dem Messgerät handelt es sich um einen Aufbau aus den 1950er Jahren. Das Laser-Photometer besteht aus einem mittels Laserlinien-Filter bei $632,8 \mathrm{~nm}$ gesäuberten Laser. Der emittierte Laserstrahl trifft auf eine Verzögerungsplatte. Diese dreht die Polarisationsrichtung des linear polarisierten Laserlichtes um einen nicht näher definierten Winkel. Über einen Umlenkspiegel strahlt dieses auf die Probe ein. Die Abbildung des erzeugten Raman-Signals erfolgt über eine Konvex-Linse auf einem Detektor. Ein Polarisations-Filter entfernt die Anregungsstrahlung. Aufgrund der verwendeten optischen Bauteile ist dieser Aufbau nicht mit dem in diesem Beitrag vorgestellten vergleichbar. In den letzten 70 Jahren ist die Entwicklung optischer Elemente durch Beschichtungsverfahren und die detektorseitige Sensitivität sowie stabilisierter, schmalbandiger Laserlichtquellen stark vorangeschritten $[4,7,8]$.

Aufbauend hierauf stellt der hier präsentierte Beitrag die Entwicklung eines aktualisierten und auf den Stand der Technik weiterentwickelten Photometers vor. Der Fokus hierbei liegt, neben dem generellen Sensorkonzept, auf einer autarken Betriebsweise in einer prozessnahen Umgebung.

\section{Material und Methoden}

In der Raman-Messtechnik sind PhotometerKonzepte bisher nicht etabliert und die meisten Patente beziehen sich nur im Ausnahmefall auf die Detektion des Raman-Effekts. Es ist bisher nur ein Gerät auf dem Markt verfügbar. Die größte Herausforderung bei der hier vorgestellten Entwicklung eines neuartigen Raman-Photometers bildet die Auswahl der Detektoren und der auf die Applikation angepassten Filter. Die Wahl fiel auf SinglePhoton-Avalanche-Diode-Detektoren (SPAD) zum Zählen von Einzelphotonen und, für eine universellere Anwendung, durchstimmbare Filter der Firma Semrock, Inc. Der Austausch dieser gegen eine fixe Filtervariante ist für das folgende Funktionsmuster vorgesehen. Da bei der Raman-Spektroskopie sehr schmale Peaks detektiert werden, ist das Filtern der Signale mit nur einem durchstimmbaren Filter nicht möglich. Die Kombination zweier bzw. mehrerer Filter ist in Abb. 1 dargestellt.

Einfallswinkel 0-60

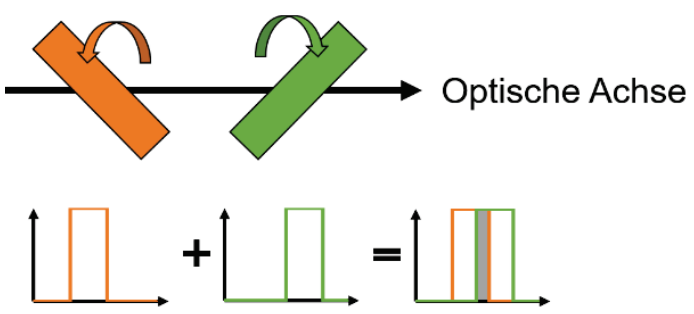

Abb. 1: Anwendungsprinzip der durchstimmbaren Filter

Durch eine geschickte Kombination von zwei Filtern, die verschieden zum optischen Strahlengang angestellt werden, besteht die Möglichkeit, bis auf ca. 80 Wellenzahlen die einfallende Strahlung zu filtern. Standard-Filter verlieren ihre Transmission in großen Teilen schon bei geringen Winkeln und sind nicht in jedem Bereich des Spektrums mit einer ausreichenden Steilheit als Massenware am Markt verfügbar.

Die Integrationszeit des Photometers betrug bei allen Messungen $1 \mathrm{~s}$ mit zehn Akkumulationen, bei den Messungen mit einem Spektrometer hinsichtlich besserer Vergleichbarkeit ebenfalls $1 \mathrm{~s}$.

\section{Ergebnisse}

Nach erfolgreicher Evaluation der Filtereinheit wurde das Gesamtsystem anhand von 
Verdünnungsreihen getestet und ein lineares Verhalten nachgewiesen.

Die photometrischen Messungen sind beispielhaft an $\mathrm{H}_{2} \mathrm{O}_{2}$ in $\mathrm{Abb}$. 2 (oben) und entsprechende spektroskopische Kontrollmessungen an einem tec5 MultiSpec $₫$ RamanSystem in Abb. 2 (unten) dargestellt.
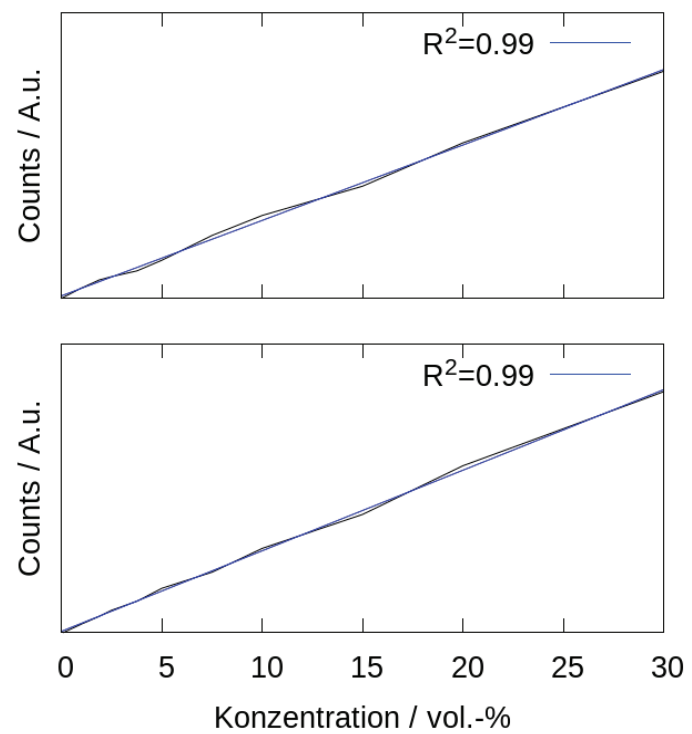

Abb. 2: $\mathrm{H}_{2} \mathrm{O}_{2}-$ Messungen mit Photometer (oben) und mit Spektrometer (unten)

Photometrische und spektroskopische Messungen weisen beide lineares Verhalten auf. Die Verfolgung von Wasserstoffperoxid ist über einen weiten Konzentrationsbereich möglich. Das Proof of Concept des Systems wurde somit erbracht und anschließend in der Reaktionsverfolgung (einer Aminwäsche) getestet.

In der chemischen Industrie ist die Bindung von $\mathrm{CO}_{2}$ aus Produkt- oder Abluftströmen von großer Bedeutung. Eine einfache und gut erforschte Methode ist die Wäsche aus gasförmigen Strömen durch Amine. Die reversible Bindung von $\mathrm{CO}_{2}$ an das Amin kann mittels Raman-Spektroskopie verfolgt werden [9]. Durch das Anpassen der Filtereinheit auf den Aminpeak bei 675 Wellenzahlen Raman-Shift ausgehend von einer Anregung bei $785 \mathrm{~nm}$ kann ein spezifischer Aminpeak zur Detektion im Photometer aus dem Spektrum "herausgefiltert" werden. Der Referenzkanal wurde direkt im angrenzenden Spektralbereich festgelegt. Die Verrechnung der beiden Kanäle findet noch im Photometer statt. Zusätzlich wird eine Dunkelstrom-Korrektur durchgeführt. Der Reaktionsverlauf ist in Abb. 3 (oben) dargestellt. Mit der Messmethode kann also die Zunahme der Amin- $\mathrm{CO}_{2}$-Bindung beobachtet werden.
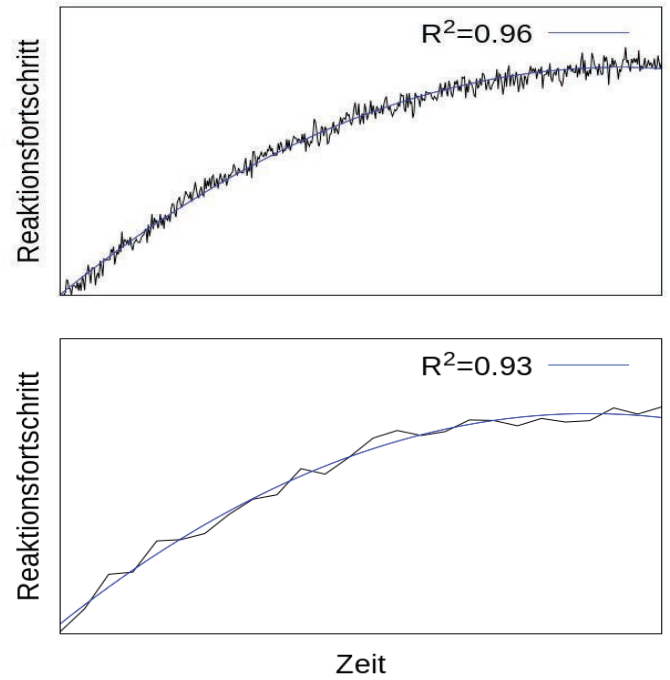

Abb. 3: Reaktionsverfolgung einer Aminwäsche mit Photometer (oben) und mit Spektrometer (unten)

Die zu Beginn der Reaktion hohe Umsatzrate verlangsamt sich im zeitlichen Verlauf und kommt letztendlich zum Erliegen. Da $\mathrm{CO}_{2}$ fein verteilt durch das Probenvolumen strömt und die Probe gerührt wird, ist das Signal durch Matrixeffekte nicht abreagierter $\mathrm{CO}_{2}$-Bläschen deutlich verrauschter, verglichen mit blasenfreien Proben. Die Messpunkte liegen sehr gut auf der entsprechenden Trendlinie. Eine Kontrollmessung erfolgte wieder mit einem tec5 AG MultiSpec $®$ Raman Spektrometer. Dies ist in Abb. 3 (unten) dargestellt.

Es ist festzuhalten, dass Reaktionen mit einem Raman-Photometer überwachbar sind. Das Vermessen von kompletten Spektren ist in dieser Applikation (Aminwäsche) nicht notwendig.

\section{Gesamtkonzept Funktionsmuster}

Viele Photometer- und Spektrometer-Systeme benötigen zur Weitergabe der erhaltenen Information an ein PLS einen zwischengeschalteten Computer und sind daher nur indirekt eingebunden. Der eingesetzte Rechner stellt zwar eine gewisse Flexibilität sicher, hat aber den intrinsischen Nachteil, dass Komponenten und Betriebssysteme aus dem Consumer-Bereich zum Einsatz kommen. Für die Verwendung im industriellen Umfeld müssen erhöhte Sicherheits- und Zuverlässigkeitsanforderungen erfüllt werden. Durch den Anstieg der Rechenleistung in Mikrokontrollern und die Verfügbarkeit von Datenverarbeitungskomponenten lässt sich die Messdatenerfassung, -verarbeitung und die Kommunikation unter Einhaltung eines industriellen Standards hiermit zuverlässiger realisieren. In das Funktionsmuster wurde eine modifizierte Version der Embedded-Plattform tecSaaS $₫$ der 
Firma tec5 AG integriert. Bislang werden hiermit optische UV/VIS/NIR Spektralsensoren als autarke Messsysteme zur Prozesskontrolle oder Qualitätsprüfung betrieben. Diese ermöglicht, Detektion, Verarbeitung und Vergleich der Messwerte mit Sollwerten sowie die Weitergabe an ein PLS in einem Gerät, basierend auf industrieller Elektronik und Firmware, zu vereinen. Das PLS nimmt demnach das Mehrkanal-Photometer als einzelnen Sensor wahr. Hier konnte das Konzept "Spectrometer as a Sensor" in einem Funktionsmuster auf eine Photometer-Anwendung („Photometer as a Sensor") übertragen werden. Während der Standardbetrieb völlig autonom erfolgt, können Parametrierung und Monitoring u.a. über eine Ethernet-Schnittstelle durchgeführt werden. Hierfür sind keine Programmierkenntnisse nötig. Kombiniert mit den inhärenten Vorteilen eines „konventionellen" Photometers, nämlich der erhöhten Robustheit durch reduzierte Komplexität, wird das Konzept zu einem Kandidaten für den Bereich der kostengünstigen und intelligenten Prozesssensorik.

Das Funktionsmuster besteht, trotz autonomer Betriebsweise, nur aus wenigen Komponenten. Diese sind in Abb. 4 dargestellt. Die gestrichelten Linien stellen den Austausch von elektrischen und die durchgezogenen von optischen Signalen dar. Embedded Plattform, Lasermodul sowie Detektionseinheit befinden sich im Photometer-Gehäuse. Die Messsonde ist über Glasfasern mit diesem verbunden.

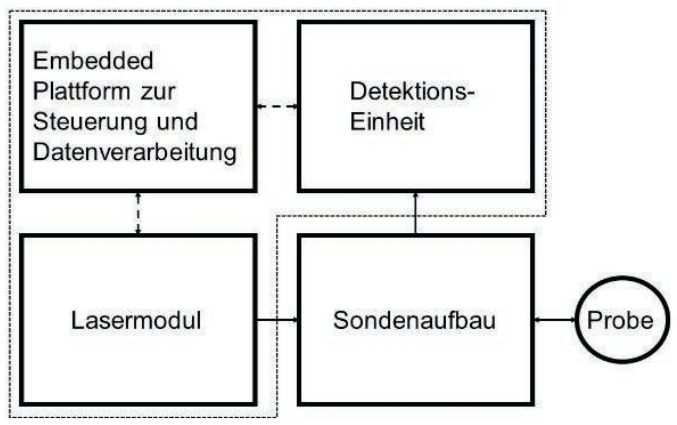

Abb. 4: Konzeptzeichnung des Funktionsmusters

Jedes zusätzliche Bauteil hat häufig einen negativen Einfluss auf die optische Ausbeute und Robustheit. Aus diesem Grund ist der optische Aufbau auf ein Minimum notwendiger Komponenten reduziert. Dies soll, neben der Minimierung von Reflexen und sonstigen optischen Verlusten, auch die Herstellkosten senken. Als Detektoren sind Multi-Pixel-PhotonCounter-Module (MPPC) der Firma Hamamatsu verbaut. Diese sind deutlich preisgünstiger als SPAD-Detektoren, sind aber ebenfalls in der Lage, Einzelphotonen zu zählen und damit ausreichend nachweisempfindlich zur Detektion von Raman-Signalen. Die Raman-Messsonde ist prinzipiell frei wählbar und nicht im Fokus dieser Arbeit. Bei dem Laser-Modul handelt es sich um einen stabilisierten $35 \mathrm{~mW}$ Laser. In Abb. 5 ist der Aufbau abgebildet.

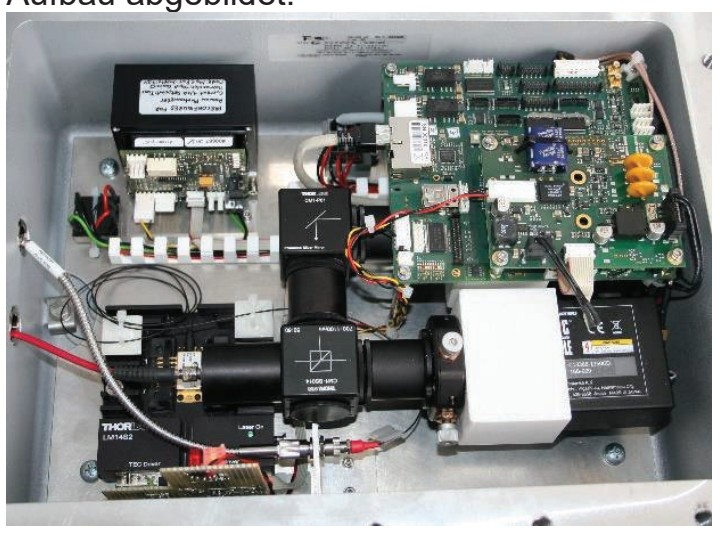

Abb. 5: Funktionsmuster in einem Ex-d-Gehäuse

In der chemischen Industrie befinden sich viele Messstellen in explosionsgefährdeten Bereichen. Um ein Gerät betreiben zu dürfen, ist die Einhaltung besonderer Vorschriften erforderlich. Für elektrische Geräte ist z.B. eine druckfeste Kapselung (Ex-d) möglich. Dies bedeutet, dass innerhalb des Gerätes erzeugte Zündfunken das Gehäuse nicht verlassen dürfen. Im Falle einer Explosion innerhalb des Gehäuses darf die freigewordene Energie das Gehäuse ebenfalls nicht schlagartig verlassen. Um dies zu gewährleisten und einen sicheren und kontinuierlichen Betrieb zu ermöglichen, befinden sich alle elektronischen Bauteile in einem druckfesten Ex-d-Gehäuse. Neben den elektrischen Gefahren geht auch eine Gefährdung durch den Eintrag von optischer Lichtleistung als mögliche Zündquelle aus. Raman-Spektrometer haben in der Prozesskontrolle die Limitierung hoher notwendiger Laserleistungen. In explosionsgeschützten Bereichen muss im Hinblick auf das Potenzial einer optischen Zündquelle der Leistungseintrag des Lasers deutlich reduziert werden. Abhängig von der Stoff- und Temperaturklasse kann die Obergrenze kleiner $35 \mathrm{~mW}$ betragen. Da die Stärke des Raman-Effekts proportional zur Anregungsleistung ist, steigt bei konventionellen Spektrometersystemen die Messzeit durch die Reduktion der Laserleistung signifikant an. Durch die gesteigerte Nachweisempfindlichkeit von Einzelphotonendetektoren, kann der erhöhte Zeitaufwand kompensiert werden. 


\section{Diskussion der Ergebnisse}

Das Design und die Konstruktion eines Ex-dfähigen Raman-Photometers konnte erfolgreich umgesetzt werden.

Die Auswahl der Messungen ergab sich aus einer Schnittmenge häufig auftretender Messaufgaben in der chemischen Industrie und der Eignung für die Applikation eines RamanPhotometers.

Der Photometer-Einsatz ist nur möglich, wenn die zu vermessenden Spektren nicht zu komplex und die einzelnen Zielmoleküle spektral deutlich unterschieden werden können. Genau dann ist der Einsatz von RamanSpektrometern nicht notwendig. Zudem zeigen Photometer die Möglichkeit, mit stark reduzierten Laserleistungen gute Signalrauschverhältnisse zu liefern, was sich auf einen Einsatz im Ex-Bereich und ebenso im Medizintechnikbereich durch deren Regulatorien positiv auswirkt.

Raman-Photometer weisen in einem stark konkurrierenden Umfeld unterschiedlicher Messstrategien je nach Anwendung deutliche Vorteile auf und werden hierbei in Zukunft eine Rolle spielen.

\section{Ausblick}

Nach der Fertigstellung des Funktionsmusters und ersten erfolgreichen Versuchen in realen Produktionsgemischen wird ein Übertrag auf chemische Anlagen im Technikumsmaßstab durchgeführt. Hierbei sind neue Herausforderungen zu meistern und das Funktionsmuster den Gegebenheiten der Prozessumgebung weiter anzupassen. Angedacht ist neben der Überwachung von chemischen Reaktionen im Batchbetrieb die Überwachung und Steuerung von kontinuierlichen Anlagen. Sollte dies gelingen, ist ein weiterer Wechsel von Technikums- zu Produktionsanlagen vorgesehen.

\section{Danksagung}

Wir bedanken uns bei Herrn Thomas Fritsch der Krohne Innovation $\mathrm{GmbH}$ für die vielen aufschlussreichen Diskussionen.

Die Projekte wurden gefördert von den Bundesministerien für Wirtschaft und Energie (Förderkennzeichen: ZF4013905NT5) und für Bildung und Forschung auf Beschluss des deutschen Bundestages (Förderkennzeichen: 13FH8I03IA).

\section{Literaturnachweis}

[1] VDI/VDE- Gesellschaft Mess- und Automatisierungstechnik, 2015. Thesen und Handlungsfelder - Technologie-Roadmap „Prozesssensoren 4.0“
[2] Schwolow, S. et al. 2015. Org. Process Res. Dev. (9), 1286-1292; doi: 10.1021/acs.oprd.5b00184

[3] Norm DIN EN 60079-28 VDE 0170-28 (201604-00)

[4] Berenblut, B. J. 1972: Journal of Physics E: Scientific Instruments, Volume 5, Number 4

[5] Brown, G. 2008: Patent US20080180661 A1

[6] Kaye, W. 1974: Patent DE000002103318B

[7] Rea, G. 1957: Abstracts of OSU International Symposium on Molecular Spectroscopy 19461959

[8] Szymanski, H. 1967: Plenum Press; doi: 001:10.1007/978-1-4684-3024-0

[9] Vogt, M. 2011: Energy Procedia 4, 1520-1525; doi: 10.1016/j.egypro.2011.02.020 